\title{
An Ultra Low-Voltage and Low-Power OTA Using Bulk-Input Technique and Its Application in Active-RC Filters
}

\author{
Arash Ahmadpour \\ Department of Electronic Engineering, Lahijan Branch, Islamic Azad University, Lahijan, Iran \\ E-mail:ar.amp@iau-lahijan.ac.ir \\ Received March 10, 2011; revised April 29, 2011; accepted May 6, 2011
}

\begin{abstract}
This paper presents the design of a two-stage bulk-input pseudo-differential operational transconductance amplifier (OTA) and its application in active-RC filters. The OTA was designed in $90 \mathrm{~nm}$ CMOS process and operates at a single supply voltage of $0.5 \mathrm{~V}$. Using a two-path bulk-driven OTA by the combination of two different amplifiers the DC gain and speed of the OTA is increased. Rail-to-rail input is made possible using the transistor's bulk terminal as in input. Also a Miller-Feed-forward (MFF) compensation is utilized which is improved the gain bandwidth (GBW) and phase margin of the OTA. In addition, a new merged cross-coupled self-cascode pair is used that can provide higher gain. Also, a novel cost-effective bulk-input common-mode feedback (CMFB) circuit has been designed. Simplicity and ability of using this new merged CMFB circuit is superior compared with state-of-the-art CMFBs. The OTA has a $70.2 \mathrm{~dB}$ DC gain, a $2.5 \mathrm{MHz}$ GBW and a $70.8^{\circ}$ phase margin for a $20 \mathrm{PF}$ capacitive load whereas consumes only $25 \mu \mathrm{w}$. Finally, an $8^{\text {th }}$ order Butterworth active Biquadrate RC filter has been designed and this OTA was checked by a typical switched-capacitor (SC) integrator with a $1 \mathrm{MHz}$ clock-frequency.
\end{abstract}

Keywords: Operational Transconductance Amplifier (OTA), Common-Mode Feedback (CMFB), Bulk-Input, Switched-Capacitor (SC) Integrator, Miller-Feed-Forward (MFF)

\section{Introduction}

There are growing strong demands for low-voltage supply and low-power consumption circuits and systems. This is especially true for very high integration level and very large scale integrated (VLSI) mixed-signal chips and system-on-a-chip. In mixed-signal systems, the ana$\log$ circuits are combined with digital circuits in order to get the best performance with low-voltage supply and low-power consumption. This combination should be in an optimal way and the optimization process is application dependent. Also modern portable applications such as medical devices and remote wireless sensors require extending the battery life as well. This trend has forced designers to develop new approaches more amenable to low-voltage and low-power integrated circuits and it poses lots of challenges for all involved such as processes, devices, circuits, and system architectures [1]. Operational transconductance amplifiers (OTAs) are the key active building blocks of continuous-time filters. They can be generally classified into single ended, fully differential and pseudo-differential OTAs. Fully differential OTAs are preferred because they provide larger signal swing, better distortion performance, better CM noise and supply noise rejection. The main drawback of using fully differential OTAs is that a common-mode feedback (CMFB) circuit must be added. The principle of the CM control circuit can be easily applied to the design of differential structures, and it is well suited for low-voltage pseudo-differential architectures. This extra circuit is used to establish the common-mode output voltage and suppress the common mode signal components [2]. The speed of the common-mode path should be comparable to that of the differential path; otherwise the common-mode noise may be significantly amplified such that the output signal becomes distorted. Also, the CMFB circuit is often a source of noise injection and increases the load capacitance that needs to be driven. Regardless of the limitations described above, fully differential OTAs work very well and can substantially im- 
prove the system's quality, especially in very unfriendly environments such as mixed-mode applications. However, at lower supply voltages, pseudo differential OTAs could be used to avoid the voltage drop across the tail current source used in the fully differential structures. Taking the tail current source achieves a larger signal swing, but it also results in larger CM gain. So it requires to carefully controlling the $\mathrm{CM}$ response for pseudo differential OTAs. The reduced supply voltage will cause many problems when designing analog circuits due to the reduction in available signal swing. This problem is magnified due to the fact that the threshold voltage $\left(V_{T}\right)$ of the transistors has been reduced at a slower rate than the supply voltage. In other words, the threshold-voltage with decreasing the Power supply never reduces linearly. If standard analog design techniques continue to be used, the dynamic range and SNR of the circuits will degrade. The gain of devices such as amplifiers will also decrease because cascode transistors can no longer be used since they limit the output swing. The design of rail-to-rail input circuits is also made more difficult due to the large $V_{T}$ which must be overcome. One solution for designing low-voltage analog circuits is to operate the transistors in the weak inversion region. The drawbacks to this technique include a limited input signal swing, an increase in the mismatch between transistors, a low slew-rate due to the low bias current levels, and large transistor sizes [3-6]. In order to avoid these drawbacks a bulk-input technique can be used, which allows for operation in the moderate inversion region at supply voltages equal to the $V_{T}$ of the technology. The voltage applied to the bulk actually reduces the threshold voltage of the transistor, which increases the inversion level. One drawback of the bulk input technique is that the input transistor must sit in an n-well or p-well, so that its bulk is separate from the bulk of the rest of the transistors. This is not a problem in advanced technologies, which make use of triple well structures. In triple-well processes both the NMOS and PMOS can have isolated bulk terminals [5]. This paper presents the design of a novel two-stage bulk-input pseudo differential OTA, which operates at a supply voltage of $0.5 \mathrm{~V}$. The circuit was designed using the $90 \mathrm{~nm}$ process in digital CMOS technology. Design procedure for this new merged OTA structure such as main OTA, CMFB and bias circuits are introduced in Section 2. In Section 3, SC integrator and active RC filter design are described. Section 4 presents simulation results. Finally, conclusions and acknowledge are given in Section 5 and 6 , respectively.

\section{Bulk-Input OTA Circuit Design}

\subsection{Main Amplifier}

A basic body-input stage, capable of operating with very low supply voltage is depicted in Figure 1. This structure is a two-path OTA built by the combination of the two different amplifiers. In this circuit a PMOS bodydriven OTA is implemented due to the action of $\mathrm{M}_{1+}$, $\mathrm{M}_{1-}, \mathrm{M}_{4+}$ and $\mathrm{M}_{4}$, and also a current-mirror bulk-input OTA is composed by transistors $\mathrm{M}_{2+}, \mathrm{M}_{2-}, \mathrm{M}_{3-}$ and $\mathrm{M}_{3+}$. The OTA input consists of two bulk-input split differential pairs; some of the ac current generated by the input transistors is injected to the output transistors, providing a fast path for the current. The other part of the current is delivered to the current mirrors of $\mathrm{M}_{3+}$ and $\mathrm{M}_{3-\text {. These }}$ are the main transistors which the main portion of the currents by the factor $n$ transfer to output transistors of $\mathrm{M}_{4+}$ and $\mathrm{M}_{4}$. If we suppose $\mathrm{M}_{4+}$ and $\mathrm{M}_{4-}$ have equal effective voltage to $\mathrm{M}_{1+}$ and $\mathrm{M}_{1-}$, their transconductances are very much greater than bulk- transconductance of $\mathrm{M}_{1+}$ and $\mathrm{M}_{1-}\left(\mathrm{g}_{\mathrm{m} 4} \gg \mathrm{g}_{\mathrm{mb} 1}\right)$. It is possible to utilize common-source amplifier which is parallel and in same phase with $M_{1}$ when the input signal is implied to the gate of $\mathrm{M}_{4}$ via feed-forward (FF) compensate capacitor $C_{f f}$ (not shown in Figure 1). Moreover, the transistor of the active load current source operates like a common source amplifier. This structure increases the gain and speed of the OTA. $\mathrm{M}_{3+}$ and $\mathrm{M}_{3-}$ with diode connections utilize for $\mathrm{M}_{4_{+}}$and $\mathrm{M}_{4}$ biasing and use in $\mathrm{M}_{2+}$ and $\mathrm{M}_{2-}$ as a differential loads.

To further improve the differential gain PMOS devices $\left(\mathrm{M}_{5+}, \mathrm{M}_{6+}\right.$ and $\left.\mathrm{M}_{5-}, \mathrm{M}_{6-}\right)$ are added. The body-inputs of them are a new merged cross-coupled self-cascode pairs that adds a negative resistance to the output in the form of $\mathrm{g}_{\mathrm{mb} 6}^{\prime}$ and boosts the differential DC gain. The $\mathrm{g}_{\mathrm{mb} 6}$ is the $\mathrm{g}_{\mathrm{mb}}$ of the self-cascode configuration of $\mathrm{M}_{5}, \mathrm{M}_{6}$. Also, the gate inputs biased at $100 \mathrm{mV}$, which biases them in moderate inversion. Moreover, these transistors are a self-cascode active load for transistors $\mathrm{M}_{4+}$ and $\mathrm{M}_{4}$ which are common source amplifiers.

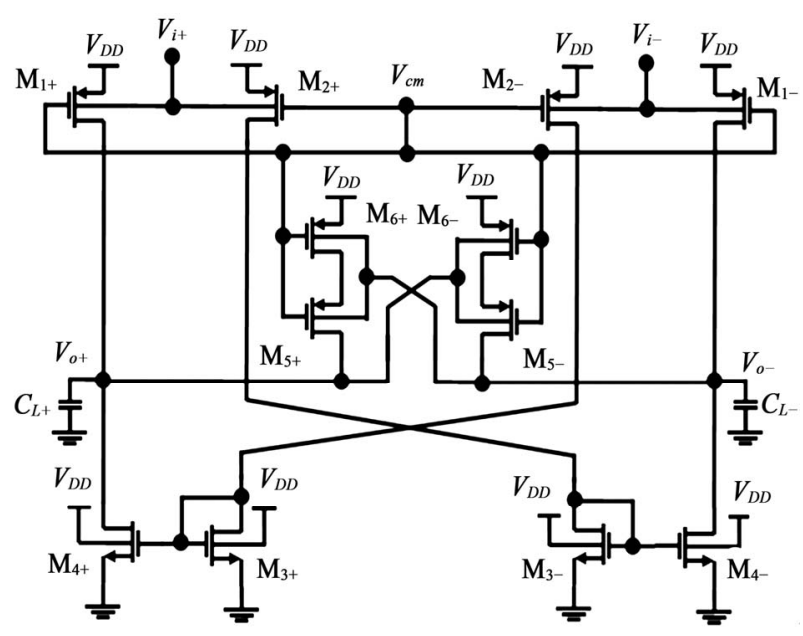

Figure 1. One stage of proposed bulk-input differential pair. 


\subsection{Bulk-Mode Common-Mode Feedback Circuit}

The Fully differential OTAs require a common-mode feedback circuit. A CMFB circuit should behave linearly and only response to common-mode voltage. Lacking these features causes increase the THD of the circuit. Nowadays, designing a CMFB circuit which is able to operate under an ultra low-voltage supply is very difficult, mainly because of the difficulty of detecting of CM voltage. In Reference [5] a CMFB circuit which operated at $0.5 \mathrm{~V}$ by using two resistors to sense the output $\mathrm{CM}$ levels was designed. But this structure increases the die area and reduces the gain due to longer loads the OTA. To overcome some of these problems, a CMFB circuit has been reported [6]. This structure uses of four PMOS and two NMOS transistors. The NMOS devices is a bulk-input current mirror, which compares the current of the PMOS devices and then difference of these current is fed to the gate of the input transistors for controlling of the output $\mathrm{CM}$ voltages. This paper is given a novel continuous-time CMFB circuit that is able to operate with an ultra low-voltage supply. Figure 2 shows the proposed CMFB circuit for each stage.

In this circuit, a combination of two complementary NMOS and PMOS transistors is used in a bulk-driven configuration. The output voltage of the CMFB circuit can be found using the KCL at the output node $\left(V_{c m}\right)$. The difference between currents of PMOS and NMOS transistors is fed to the gate of the input transistors of OTA. The bulks of the PMOS devices $\mathrm{M}_{\mathrm{cp}+}\left(\mathrm{M}_{\mathrm{cp}-}\right)$ connected to the NMOS devices $\mathrm{M}_{\mathrm{cn}+}\left(\mathrm{M}_{\mathrm{cn}-}\right)$ and are utilized to maintain the output common voltage at the required level $(250 \mathrm{mV})$, while maximizing the output swing of the OTA. In other words, the inputs to the CMFB circuit are the outputs of the OTA, $V_{o^{+}}$and $V_{o^{-}}$. The CMFB circuit amplifies the difference between the average of $V_{o^{+}}$ and $V_{o^{-}}$and the desired common level $V_{c m}(250 \mathrm{mV})$, and sends a feedback signal $V_{c m}$ to set the bias voltage at the gates of input transistors of the OTA. This structure is able to operate with an ultra low supply voltage (as low as $0.3 \mathrm{~V}$ supply voltage).

In addition, this configuration with minimizing of the area cost and power consumption is a very cost-effective CMFB which is much more applicable regarding modern ultra low-voltage and low-power mixed-signal SoCs. Simplicity and ability of using this new merged CMFB circuit to set the output CM voltage of OTA is superior compared with state-of-the-art CMFB.

To obtain adequate gain, identical gain blocks can be cascaded so that a two-stage OTA is obtained. Figure 3 show the proposed OTA structure in first and second stages without bias and CMFB circuits. In order to improve the phase margin and speed of amplifier, we were used Feed-forward compensation. Finally, the OTA was stabilized by adding Miller compensation capacitors $\mathrm{Cc}$ with series resistors Rc for right half-plane zero cancelation. The OTA was designed and classified into two kinds of compensations, such as only Miller compensation and Miller-Feed-forward (MFF) compensation. The comparison of two these methods of compensations are given in Table 1. As can be seen, the MFF compensation method increases the phase margin and enhances the open-loop GBW about two times as much.

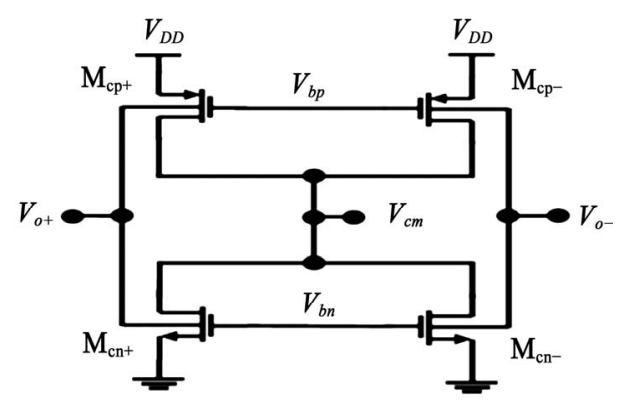

Figure 2. Ultra low-voltage bulk-mode CMFB circuit.

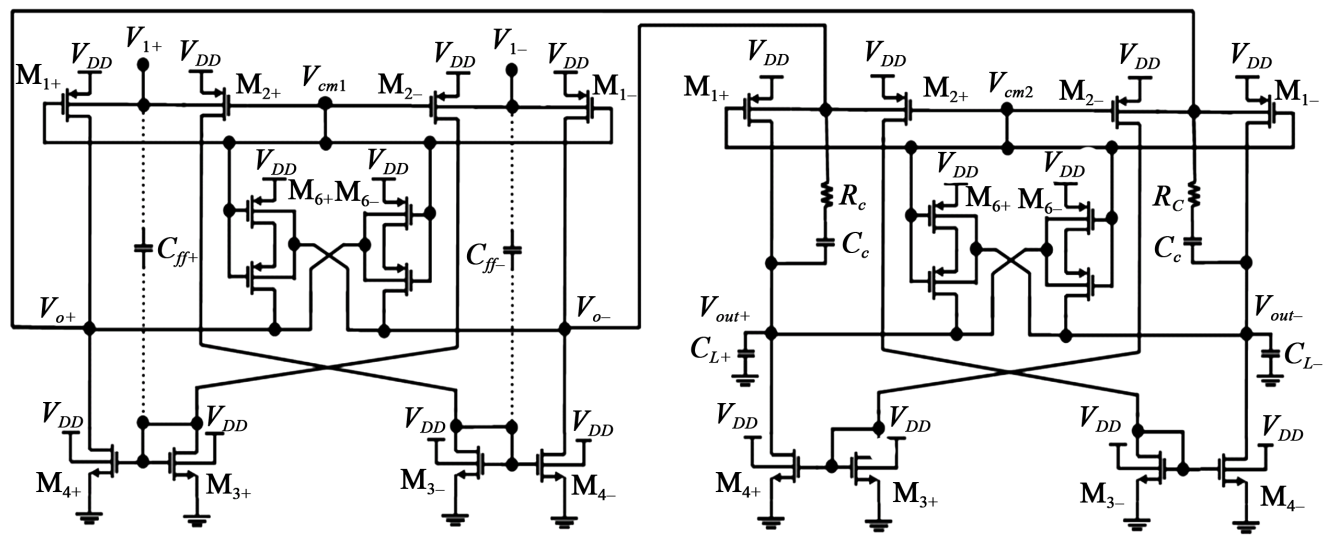

Figure 3. Two-stage bulk-input pseudo-differential OTA without bias and CMFB circuits. 
Table 1. Comparison of two compensation methods.

\begin{tabular}{ccc}
\hline Resistors and Capacitors & Miller-comp. & MFF-comp. \\
\hline$R_{c}$ & $60 \mathrm{k}$ & $68 \mathrm{k}$ \\
$C_{c}$ & $3 \mathrm{pf}$ & $1 \mathrm{pf}$ \\
$C_{f f}$ & - & $3 \mathrm{pf}$ \\
Open-loop GBW & $962 \mathrm{KHz}$ & $2.5 \mathrm{MHz}$ \\
Phase-Margin & $65^{\circ}$ & $70.8^{\circ}$ \\
\hline
\end{tabular}

\subsection{Reference Current Generator and Bias Circuit}

A low-sensitivity reference current generator and bias circuit are illustrated in Figure 4. Because the gate and source of $M_{B 3}$ and $M_{B 4}$ are common for both transistors, and the aspect ratios are equal, $I_{D M B 3}=I_{D M B 4}$. Also, note that $V_{G S M B 2}=V_{G S M B 1}+R_{B} \cdot I_{D M B 1}$. Thus,

$$
\begin{aligned}
& \sqrt{\frac{2 I_{D M_{B 3}}}{\mu_{n} \cdot C_{o x} \cdot(W / L)_{M_{B 2}}}} \\
= & \sqrt{\frac{2 I_{D M_{B 3}}}{\mu_{n} \cdot C_{o x} \cdot K \cdot(W / L)_{M_{B 2}}}}+R_{B} \cdot I_{D M_{B 3}}
\end{aligned}
$$

In the above mentioned equation, $K$ is the ratio between the aspect ratios of $M_{B 1}$ and $M_{B 2}$. Rearranging this expression,

$$
I_{D M_{B 3}}=\frac{1}{R_{B}^{2}} \frac{2}{\mu_{n} \cdot C_{o x} \cdot K \cdot(W / L)_{M_{B 2}}}\left(1-\frac{1}{K}\right)
$$

In the target circuit, $k=1.13$ and $R_{B}=1 \mathrm{k}$, thus $I_{D M B 3}$ $=1.25 \mathrm{uA}$. As expected, the circuit is independent of the supply voltage. Transistor $M_{B 5}$ mirrors this current to generate a stable $2 \mathrm{uA}$ reference current, which is used in the bias of PMOS devices. In order to ensure that the all transistors operate in the saturation region, fixed bias voltages are applied either to the gate of the PMOS and NMOS transistors in the main OTA and CMFB circuits. The gate voltage all of the NMOS and PMOS transistors biased in about $V_{b n}=350 \mathrm{mV}$ and $V_{b p}=100 \mathrm{mV}$, respectively.

\section{SC Integrator and Active Filter Circuit}

In order to show that the designed OTA will be useful in practical analog circuits, this structure was checked by a typical switched-capacitor (SC) integrator $[7,8]$ with suitable input frequency $1 \mathrm{KHz}$ and sampling frequency $1 \mathrm{MHz}$. Figure 5 is shown schematic of this structure. In switched-capacitor integrator design, we utilized two clock-frequencies which have no overlap and used Bootstrapped switches in Reference [9].

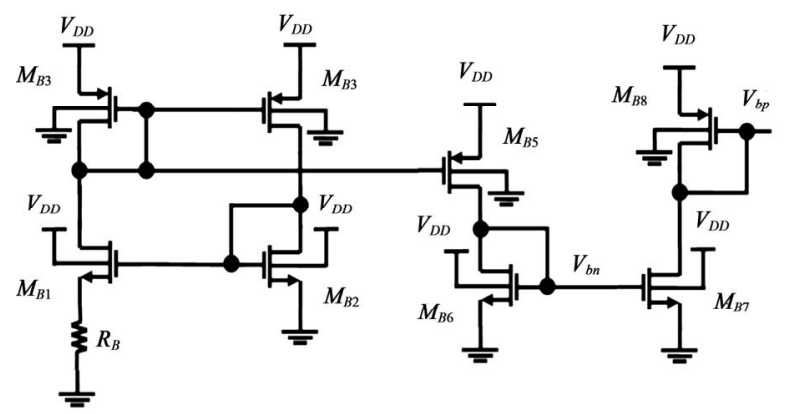

Figure 4. Reference current generator and bias circuit.

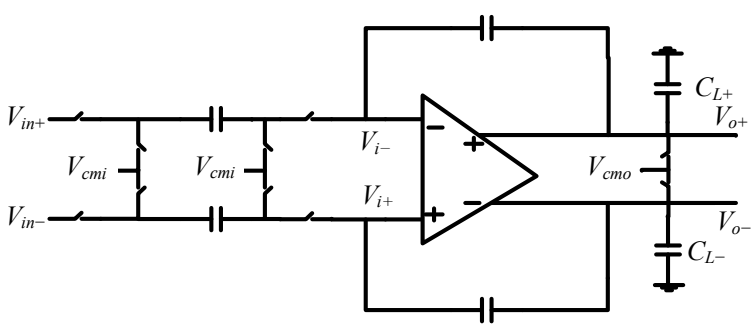

Figure 5. Fully differential switched-capacitor integrato.

Simulation result of this structure is depicted in Figure 11.

Filters are systems that can be used to manipulate the frequency spectrum of signals and they are essential in many different applications. The low-pass continuoustime filter is used to limit signal and noise bandwidth and provide anti-aliasing prior sampling. The analog-to- digital converter (ADC) digitizes the filtered output to take the advantages of the Digital Signal Processing (DSP) unit. The equalizer provides the equalization and the equalized signal goes to the decoder. The gain/timing control module is used to adjust the gain of the VGA. Although we are living in a digital age, many digital systems interfacing with the real analog world might use continuous-time filters. There are mainly two types of filters: digital filters and analog filters. While the data samples are discrete for digital filters, analog filters process continuous signals. Analog filters can be further divided into passive filters and active filters. While passive filters comprise passive components only such as resistors, capacitors, and inductors, active filters use active devices such as operational amplifiers and/or operational transconductance amplifiers (OTAs). Active filters can also be classified into Active-RC, Switched-Ca- pacitor (sampled-data filters), OTA-C/Gm-C, and LC filters. Passive filters do not employ amplifiers and usually they are off-chip filters and are not suitable for integrated circuits. But active-RC and SC filters are suitable for low to medium frequency applications. 
This OTA was tested in an $8^{\text {th }}$ order active Biquadrate $\mathrm{RC}$ filter for maximize the attainable swing. The filter was built by connecting four $2^{\text {nd }}$ orders Thomas BiQuad stage to implement the Butterworth function, shown in Figure 6. The equations of the Two-Thomas biquad filter as follow:

$$
\omega_{\circ}^{2}=\frac{1}{R_{s 2} \cdot R_{f 2} \cdot C_{f 1} \cdot C_{f 2}}, Q=\frac{R_{f 1}}{\sqrt{R_{s 2} \cdot R_{f 2}}} \sqrt{\frac{C_{f 1}}{C_{f 2}}}, A_{\circ}=\frac{R_{f 2}}{R_{s 1}}
$$

where $A_{0}$ is the gain of integrator and $\omega_{\circ}$ and $\mathrm{Q}$ are the cut-off frequency and quality factor of integrator, respectively. Assume $R_{s 2}=R_{f 2}, C_{f 1}=C_{f 2}$, Equation (3) can be written as:

$$
\omega_{\circ}=\frac{1}{R_{f 2} \cdot C_{f 1}}, Q=\frac{R_{f 1}}{R_{f 2}}, A_{\circ}=\frac{R_{f 2}}{R_{s 1}}
$$

In the above mentioned equation, $\mathrm{Q}$ and $A_{\circ}$ are determined by the ratio of resistors and both $\mathrm{Q}$ and $\omega$ 。 can be tuned independently [10]. This $8^{\text {th }}$ order Butterworth filter consists of four cascaded Thomas biquads; and the cut-off frequency is about $400 \mathrm{KHz}$. The filter was simulated with components values given in Table 2. Also, a plot of the frequency response for input $\mathrm{CM}$ voltage from rail-to-rail of this filter is shown in Figure 12.

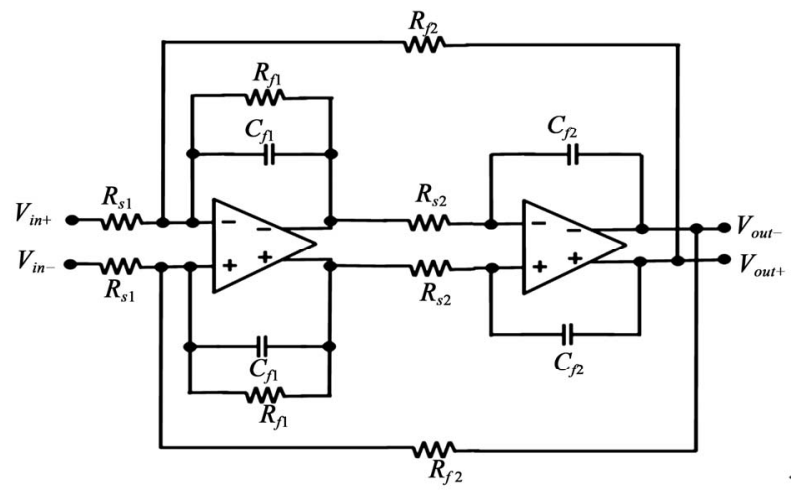

Figure 6. $2^{\text {nd }}$ order Thomas Biquad filter stage.

Table 2. Filter component values.

\begin{tabular}{ccccc}
\hline Components & Stage (1) & Stage (2) & Stage (3) & Stage (4) \\
\hline$R_{s 1}$ & $56 \mathrm{k}$ & $39 \mathrm{k}$ & $47 \mathrm{k}$ & $56 \mathrm{k}$ \\
$R_{s 2}$ & $56 \mathrm{k}$ & $39 \mathrm{k}$ & $47 \mathrm{k}$ & $56 \mathrm{k}$ \\
$R_{f 1}$ & $30 \mathrm{k}$ & $87 \mathrm{k}$ & $87 \mathrm{k}$ & $30 \mathrm{k}$ \\
$R_{f 2}$ & $56 \mathrm{k}$ & $39 \mathrm{k}$ & $47 \mathrm{k}$ & $56 \mathrm{k}$ \\
$C_{f 1}$ & $10 \mathrm{pf}$ & $10 \mathrm{pf}$ & $10 \mathrm{pf}$ & $12 \mathrm{pf}$ \\
$C_{f 2}$ & $10 \mathrm{pf}$ & $8 \mathrm{pf}$ & $5.4 \mathrm{pf}$ & $10 \mathrm{pf}$ \\
\hline
\end{tabular}

\section{Simulation Results}

To test op-amp's performance, various configurations were implemented to simulate application condition. Based on the analysis procedure described in the previous sections, an ultra low-voltage OTA at a single $0.5 \mathrm{~V}$ supply voltage was designed. The OTA has been designed in $90 \mathrm{~nm}$ CMOS process, and then simulated by Hspice. Then this structure was checked by a typical SC integrator with a $1 \mathrm{MHz}$ clock frequency. Also a 8th order active RC-filter with a $400 \mathrm{KHz}$ cut-off frequency was designed. The simulation results are shown in Figures 7 to 12. Furthermore, Table 3 summarizes the simulated performance of this OTA and comparisons of characteristics of proposed OTA with state-of-the-art OTAs. The open-loop frequency response of the OTA for the common-mode input $V_{c m}$ varying from rail-to-rail by a step $0.01 \mathrm{~V}$ was tested. The amplitudes and the phases of the proposed OTA were almost independent of the applied $V_{c m}$. Figure 5 shows the frequency response of the OTA for $V_{c m}=$ $0.25 \mathrm{~V}$. The result showed a $70.2 \mathrm{~dB}$ DC gain, a $2.5 \mathrm{MHz}$ bandwidth and a of $70.8^{\circ}$ phase margin. For the closed-loop simulation, the OTA was configured in unity-gain with $1 \mathrm{pf}$ capacitors.

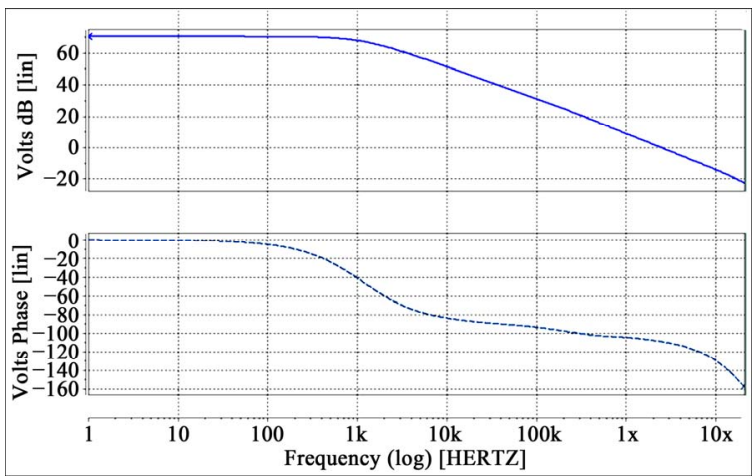

Figure 7. Open-loop frequency response of OTA for $V_{c m}=$ $0.25 \mathrm{~V}$.

Table 3. Comparison of characteristics of proposed OTA with state-of-the-art OTAs.

\begin{tabular}{lccc}
\hline \multicolumn{1}{c}{ Parameters } & This work & Ref. [5] & Ref. [6] \\
\hline Technology & $90 \mathrm{~nm}$ & $0.18 \mu \mathrm{m}$ & $0.18 \mu \mathrm{m}$ \\
Supply Voltage & $0.5 \mathrm{~V}$ & $0.5 \mathrm{~V}$ & $0.5 \mathrm{~V}$ \\
Open-loop DC Gain & $70.2 \mathrm{~dB}$ & $62 \mathrm{~dB}$ & $65 \mathrm{~dB}$ \\
Open-loop GBW & $2.5 \mathrm{MHz}$ & $10 \mathrm{MHz}$ & $550 \mathrm{KHz}$ \\
Closed-loop GBW & $645 \mathrm{KHz}$ & - & $475 \mathrm{KHz}$ \\
Phase-Margin & $70.8^{\circ}$ & $60^{\circ}$ & $50^{\circ}$ \\
Input ref. noise@1 & $139 \mathrm{nV} / \mathrm{Hz}^{1 / 2}$ & - & 432 \\
KHz & 100 & 133.3 & $\mathrm{nV} / \mathrm{Hz}^{1 / 2}$ \\
FOM & $0.119 \%$ & $1 \%$ & $0.13 \%$ \\
THD@ 500 mV & $20 \mathrm{pf}$ & $20 \mathrm{pf}$ & $20 \mathrm{pf}$ \\
Load Capacitors & $25 \mu \mathrm{w}$ & $110 \mu \mathrm{w}$ & $28 \mu \mathrm{w}$ \\
Power Consumption & \multicolumn{3}{c}{} \\
\hline
\end{tabular}


The closed-loop frequency response of the OTA is shown in Figure 8 for rail-to-rail input common-mod ranges by a step of $50 \mathrm{mV}$. It is obvious that the frequency responses of OTA have no peak and result a bandwidth of $645 \mathrm{KHz}$. This shows that the OTA function correctly for rail-to-rail input common-mode voltage values without any considerable decrease in gain.

The main optimization steps were done in transient tests because it is most important behavior. Two inputs were applied to see both small and large signal behavior of OTA in transient mode, when in closed-loop test; the OTA is configured in unity-gain with $1 \mathrm{pf}$ capacitors. For an input common-mode voltage of $250 \mathrm{mV}$, when $0.1 \mathrm{~V}$ step was applied to inputs of OTA, the output voltage was settled to its final value in less than 735 ns (@) $0.01 \%$ ). Identically, for large signal mode with a $0.3 \mathrm{~V}$ step and input common-mode voltage of $250 \mathrm{mV}$, output voltage was settled in less than $1500 \mathrm{~ns}$ for rising and less than 1170 ns for falling (@0.02\%).

In this case, positive and negative slew-rates are $0.67 \mathrm{~V} / \mu \mathrm{s}$ and $0.8 \mathrm{~V} / \mu \mathrm{s}$, respectively. Figure 9 show tran-

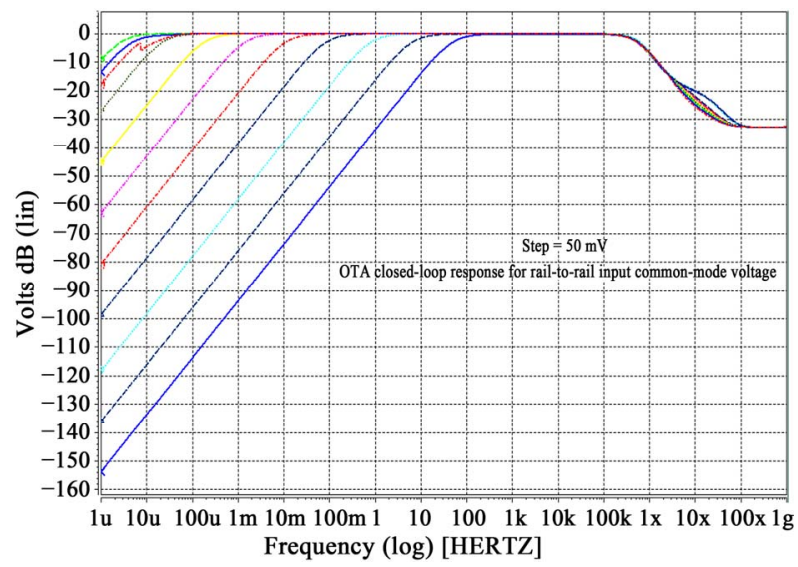

Figure 8. OTA closed-loop response for rail-to-rail common mode input value $($ Step $=50 \mathrm{mV})$.

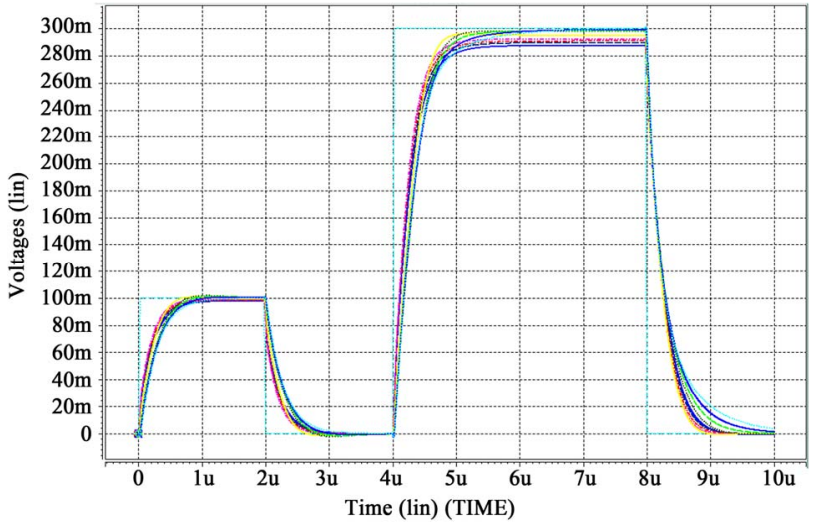

Figure 9. Settling simulated results of OTA for rail-to-rail input common-mode voltage $($ Step $=50 \mathrm{mV})$. sient response of OTA for $0.1 \mathrm{~V}$ and $0.3 \mathrm{~V}$ inputs, when input common-mode voltage varying from rail-to-rail by a step $50 \mathrm{mV}$. It is obvious that response has no ring or overshoot because of suitable bandwidth and phase margin.

The obtained total harmonic distortion of OTA with a $200 \mathrm{mV}$ amplitude and $10 \mathrm{KHz}$ input frequency sampled at $100 \mathrm{KHz}$ is shown in Figure 10. The third harmonic is

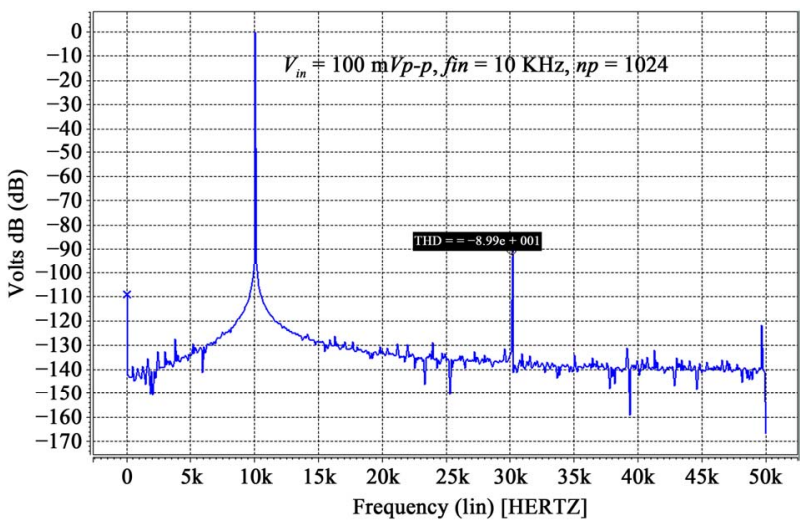

Figure 10. Total harmonic distortion of OTA with $f_{\text {in }}=$ $10 \mathrm{KHz}$ and $V_{\text {in }}=200 \mathrm{mVp}-\mathrm{p}$.

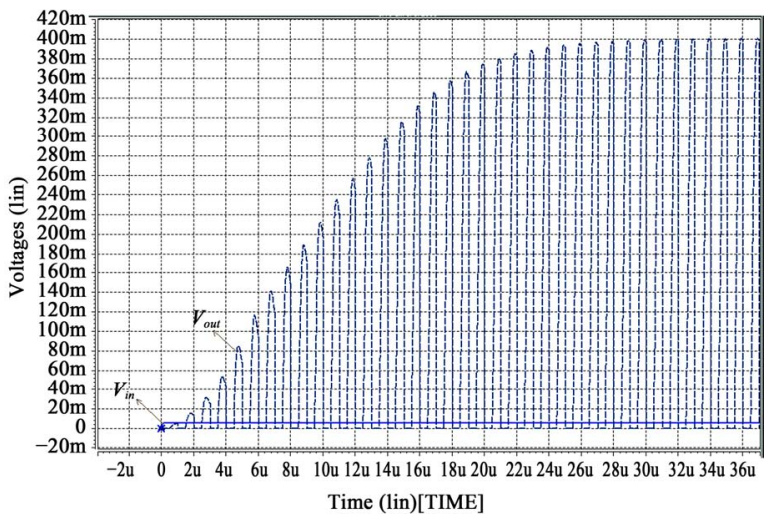

Figure 11. Integrated output voltage of OTA with $\boldsymbol{f}_{s}=1 \mathrm{MHz}$ and $f_{\text {in }}=1 \mathrm{KHz}$.

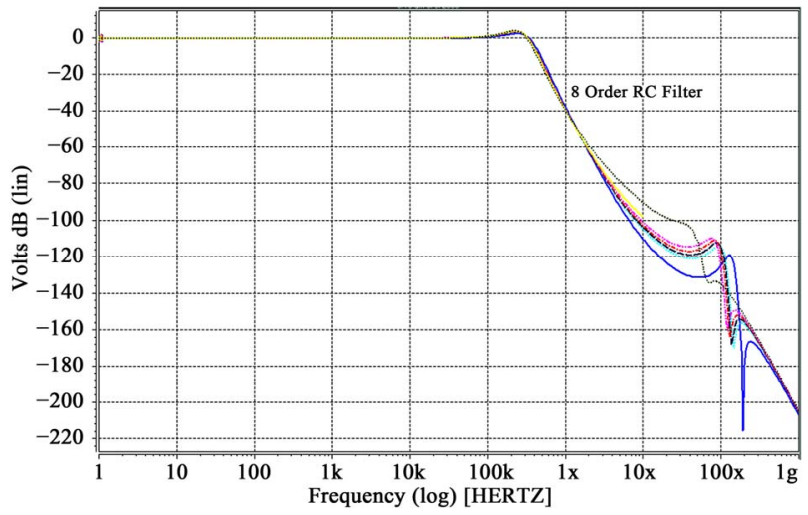

Figure 12. Filter frequency response of OTA v. $s$ input CM voltage from rail-to-rail. 
about $90 \mathrm{~dB}$ below the fundamental. Also, for $400 \mathrm{mV}$ amplitude, the third harmonic is about $-74.4 \mathrm{~dB}$. As can be seen, the extra harmonics except main harmonic has been eliminated in this design.

\section{Conclusions}

In this paper a novel two-stage configuration of the ultra low-voltage and low-power bulk-input CMOS OTA in $90 \mathrm{~nm}$ CMOS process which is able to operate with a single supply voltage as low as $0.5 \mathrm{~V}$ has been presented. A new merged cross-coupled self-cascode pair was used and higher DC gain was achieved. In addition, a MFF compensation was utilized which has been improved the GBW and phase margin of the OTA. Also, a new bulkmode CMFB circuit which no longer loads OTA has been discussed. A large linear signal swing has been achieved due to the well controlled CM behavior. Finally this structure was checked by a typical SC integrator and was tested in an 8th order active Biquadrate RC filter. Correct functionality for this configuration is verified from $-30^{\circ} \mathrm{C}$ to $70^{\circ} \mathrm{C}$. In addition, this structure can be used for modern ultra low-voltage and low-power mixed- signal SoCs.

\section{Acknowledgements}

The work described in this paper is financially supported by a grant from the Research department of the Islamic Azad University-Lahijan Branch. Also, the corresponding author wishes to thank Reviewers for their useful comments and suggestions.

\section{References}

[1] E. Sanchez-Sinencio and A. G. Andreou, "Low-Voltage and Low Power Integrated Circuits and Systems," LowVoltage Mixed-Signal Circuits, IEEE Press, New York,
1998.

[2] A. N. Mohieldin, "High Performance Continuous-Time Filters for Information Transfer Systems," Ph.D. Dissertation, Department of Electrical Engineering, Texas A \& M University, College Station, 2003.

[3] S. Yan and Sanchez-Sinencio, "Low-Voltage Analog Circuit Design Techniques: A Tutorial," IEICE Transactions on Analog Integrated Circuits and Systems, Vol. E00-A, No. 2, 2000, pp. 179-196.

[4] S. S. Rajput and S. S. Jamuar, "Low-Voltage Analog Circuit Design Techniques," IEEE Circuit and System Magazine, Vol. 2, No. 1, 2002, pp. 24-42.

[5] S. Chatterjee, Y. Tsvidis and P. Kinget, "0.5 V Analog Circuit Techniques and Their Application to OTA and Filter Design," IEEE Journal of Solid State Circuits, Vol. 40, No. 12, 2005, pp. 2373-2387. doi:10.1109/JSSC.2005.856280

[6] M. Trakimas and S. Sonkusale, "A $0.5 \mathrm{~V}$ Bulk-Input OTA with Improved Common-Mode Feedback for Low-Frequency Filtering Applications," Analog Integrated Circuits and Signal Processing, Springer Press, New York, 2009, pp. 83-89.

[7] A. Ahmadpour and R. Fouladi, "A Modified High-Performance Structure of Low-Voltage CMOS Op-Amp," Proceeding of the 2008 IEEE International Conference on Electron Devices and Solid-State Circuits, Hong Kong, 8-10 December 2008, pp. 1-5. doi:10.1109/EDSSC.2008.4760687

[8] A. Ahmadpour, "An Ultra Low-Voltage and High-Speed Amplifier for Switched-Capacitor Applications," 6th International Conference on Electrical Engineering/Electronics, Computer, Telecommunications and Information Technology, Vol. 1, Pattaya, 6-9 May 2009, pp. 552-555.

[9] M. Dessouky and A. Kaiser, "Very Low-Voltage Digital-Audio Modulator with 88-dB Dynamic Range Using Local Switch Bootstrapping," IEEE Journal of Solid-State Circuits, Vol. 36, No. 3, March 2001, pp. 349355. doi:10.1109/4.910473

[10] R. Schaumann and M. E. Van Valkenburg, "Design of Analog Filters,” Oxford University Press, Oxford, 2001. 